\title{
Feedback control of linear systems with optimal sensor and actuator selection
}

\author{
Taranjitsingh Singh ${ }^{1,2}$, Massimo De Mauri ${ }^{1,2}$, Wilm Decré ${ }^{1,2}$, Jan Swevers ${ }^{1,2}$ and Goele \\ Pipeleers $^{1,2}$
}

\begin{abstract}
This article demonstrates a combined $\mathcal{H}_{\infty}$ feedback control design for linear time-invariant (LTI) and linear parametervarying (LPV) systems and optimal sensors and actuators selection. The combined design problem is systematically constructed as a Mixed Boolean Semi-Definite Programming (MBSDP) optimization problem. We impose Big-M reformulations to the NP-hard coupled problem to be solved as a convex optimization problem using Branch and Bound (BNB) Algorithm. The combined design of dynamic output feedback control along with optimal actuator selection for an LTI seismic rejection controller design serves as an application for validation by simulation. Additionally, active vibration control of a smart composite plate along with optimal sensor and actuator selection validates the developed approach for LPV controller synthesis. On comparing this approach with exhaustive search (ES), it is observed that MBSDP approaches has faster computation time and comparing with iterative re-weighted $\ell_{1}$ norm algorithm (IRLA) and MBSDP using Outer Approximations (OA), MBSDP yields a global solution.
\end{abstract}

\section{Keywords}

$\mathcal{H}_{\infty}$ feedback control, sensor and actuator selection, active vibration control of a smart structure, seismic isolation of a large building

\section{Nomenclature}

$0_{n \times n} \quad$ Matrix of dimension $n \times n$ with all its entries 0

$1_{n \times n} \quad$ Matrix of dimension $n \times n$ with all its entries 1. The subscripts are not mentioned when the dimensions are understood from the context.

$\begin{array}{ll}\mathbb{R}^{m \times n} & \text { Set of real matrices of dimension } m \times n \\ \mathbb{R}^{n} & \text { Set of real vectors of dimension } n \\ \mathcal{X} & X \text { for LTI and } X(\rho(t)) \text { for LPV systems } \\ \star & \text { A symmetric term in any LMI inequality } \\ \operatorname{Tr}(X) & \text { Trace of the matrix } X \\ H e\{X\} & \text { Sum of the matrix and its transpose, } X+X^{T} \\ I_{n} & \text { Identity matrix of dimension } n \times n \\ X(\rho(t)) & \text { A real continuous function of } \rho(t) \\ X \prec 0 & \text { Negative-definiteness of a symmetric matrix } \\ X \succ 0 & \text { Positive-definiteness of a symmetric matrix } \\ X^{T} & \text { Transpose of the matrix } X\end{array}$

\section{Introduction}

Industries demand stable and high-performance feedback controllers to deal with multi-input multi-output (MIMO) systems. This accounts for designing a stable feedback controller $\mathcal{K}$ that satisfies the required closed-loop performance specifications for a mathematical model $\mathcal{G}$ portraying the dynamical system behaviour. Optimal $\mathcal{H}_{\infty}$ dynamic output feedback control design is widely favored to obtain $\mathcal{K}$ due to its accountability of the systems' complex dynamic behavior. Firstly, researchers Gahinet and Apkarian (1994) developed $\mathcal{H}_{\infty}$ methods using linear matrix inequalities (LMIs) for linear time-invariant (LTI) systems. Later, researchers Becker and Packard (1994) extended these techniques for linear parameter-varying (LPV) systems, a class of nonlinear systems which can be characterized using linear model and which depends on real-time measurable parameters.

A feedback control design is typically performed for a given set of actuators and sensors. If these sets are not fixed in advanced, finding the optimal controller should be combined with finding the best set of actuators and sensors, such that the best trade-off between closed-loop performance and lowest implementation cost (as few sensors and actuators as possible) can be found systematically and efficiently. In general, if there are $n_{s}$ sensors and $n_{a}$ actuators, then the possible number of combinations are $\left(2^{n_{a}}-1\right)\left(2^{n_{s}}-1\right)+$ 1. Thus, in order to find the best combination that achieves desired closed-loop performance, a computationally more affordable and low-cost methodology is required.

Researchers in Van De Wal and De Jager (2001) discussed various controller-independent strategies, Gawronski (2004)

\footnotetext{
${ }^{1}$ Department of Mechanical Engineering, Div. RAM, KU Leuven

${ }^{2}$ DMMS lab, Flanders Make

\section{Corresponding author:}

Taranjitsingh Singh

KU Leuven, Celestijnenlaan 300B, B-3001, Heverlee, Leuven

Email: taranjitsingh.singh@kuleuven.be
} 
and Nestorović and Trajkov (2013) used open-loop $\mathcal{H}_{\infty} / \mathcal{H}_{2}$ norms, and Summers et al. (2015) considered the calculations of observability and controllability gramians for the selection of sensors and actuators. Although these approaches perform well for a control design after the selection based on open loop, they do not consider this selection concerning closed-loop performance. A controllerdependent strategy like combined linear quadratic regulator (LQR) design and sensor/actuator selection using a heuristic techniques like genetic algorithm is discussed and studied in Hiramoto et al. (2000) and Haemers et al. (2019) for active suspension control of the vehicles. The main drawbacks of this technique are that they cannot guarantee a globally optimal solution and does not consider model uncertainties, hence controller robustness cannot be accounted for.

Another heuristic technique for combining control design with optimal selection is to explore sparsity in controller matrices of the controller using $\ell_{0}$-quasi norms. In order to solve these combinatorial problems, convex relaxations of $\ell_{0}$ norms as weighted $\ell_{1}$ norms are deployed in Tropp (2006) and Boyd and Vandenberghe (2004). Dhingra et al. (2014) and Argha et al. (2017) used this technique for sparse $\mathcal{H}_{\infty} / \mathcal{H}_{2}$ state feedback (SF) and static-output feedback (SOF) controller design. This heuristic approach is extended to $\mathcal{H}_{\infty}$ dynamic output feedback control design in Singh et al. (2018) by exploring sparsity in the controller matrices of the controller. Argha et al. (2019) presented a similar approach with conservative LMIs for the controller synthesis. However, in general, these heuristics techniques may not attain globally solution due to relaxations of $\ell_{0}$ norms.

The selection of a sensor or actuator may also be modeled with a boolean decision variable. Combining boolean variables with the semi-definite variables of the controller synthesis yield mixed boolean semi-definite variables, and eventually the combined $\mathcal{H}_{\infty}$ control design with optimal selection leads to mixed boolean semi-definite programming (MBSDP) problem. De Oliveira and Geromel (2000) and Hiramoto et al. (2009) separated these coupling of the mixed terms by following a two-step approach: first solving for sensors and then fixing the solution and solving for actuator selection. Finding a globally optimal solution cannot be guaranteed with this two-step approach.

This paper presents a method to separate the MBSDP terms using Big-M reformulations to solve the combined problem of $\mathcal{H}_{\infty}$ optimal dynamic output feedback controller design and optimal sensor/actuator selection as a convex optimization problem. The same approach was adopted in Nugroho et al. (2019), however, they design a stabilizing SOF controller and do not consider closedloop performances. In general, SOF is a non-convex rank minimization optimization problem of a dynamic output feedback controller synthesis. It is solved imposing assumptions on the structure of the output matrix of plant, refer Sadabadi and Peaucelle (2016) for the propositions and assumptions. Researchers Crusius and Trofino (1999) and Rubió-Massegú et al. (2013) solved the LMIs without the rank constraints by considering conservative approaches. However, this approach involves additional equality constraints, which do not represent all stabilizing SOF controllers Goßmann and Svaricek (2019).
This article tackles the combined design problem for LTI and LPV control design with optimal sensor and actuator selection. Here, the boolean and the SDP variables are decoupled using Big-M reformulations which were discussed in our previous work Singh et al. (2018) for LTI controller synthesis and were introduced in Singh et al. (2019) for LPV controller synthesis. We formulate the combined design problem by representing the LMIs generalized for LTI and LPV controller synthesis as an optimization problem. Unlike LTI, the Big-M constraints on LPV controller synthesis are enforced on the coefficients of the B-splines of the controller variables Hilhorst et al. (2016). The final convex problem is solved using classical Branch and Bound (MBSDP-BNB) algorithm Singh et al. (2018). Additionally, our main contribution lies in applying and validating this approach to LTI and LPV case studies in applications. The LPV combined control design in our article is applied to the optimal sensor and actuator selection. This is an extension of our previous work Singh et al. (2019), where the combined control design was applied with only actuator selection for a composite plate.

The rest of the article is organized as follows. Section. 2 briefly describes the $\mathcal{H}_{\infty}$ controller synthesis generalized for LTI and LPV systems. The LPV controller synthesis is based on the novel B-splines. Section. 3 combines the $\mathcal{H}_{\infty}$ control design problem with the optimal sensor and actuator selection and formulates the problem as an optimization problem. Here, the Big-M reformulations are imposed on the controller variables (LTI) or the coefficients of controller matrices (LPV) to provide a convex optimization problem which is solved by BNB algorithm. In section. 4, the mentioned approach is applied to seismic vibration control of a 20-story building for LTI controller synthesis and active vibration control of a composite plate for an LPV controller synthesis. In section 5, a comparison study is carried out between different approaches available, that are Iterative Reweighted $\ell_{1}$-norm Algorithm (IRLA) Singh et al. (2018), MBSDP-OA Coey et al. (2018) and Exhaustive Search (ES). Discussions on comparison of methods and limitations are also carried out in this section. Finally, section. 6 concludes the article.

\section{$2 \mathcal{H}_{\infty}$ controller design}

Assume a continuous-time generalized plant $\mathcal{P}$ expressed as follows:

$$
\mathcal{P}:\left\{\begin{array}{l}
\dot{x}(t)=\mathcal{A} x(t)+\mathcal{B}_{w} w(t)+\mathcal{B}_{u} u(t) \\
z(t)=\mathcal{C}_{z} x(t)+\mathcal{D}_{z w} w(t)+\mathcal{D}_{z u} u(t) \\
y(t)=\mathcal{C}_{y} x(t)+\mathcal{D}_{y w} w(t)
\end{array}\right.
$$

where $x \in \mathbb{R}^{n_{x}}, w \in \mathbb{R}^{n_{w}}, u \in \mathbb{R}^{n_{u}}, z \in \mathbb{R}^{n_{z}}$ and $y \in \mathbb{R}^{n_{y}}$ represents the plant state vector, the exogenous input, the control input, the regulated output and the measured output signals respectively. For a MIMO system, the matrix $\mathcal{A} \in$ $\mathbb{R}^{n_{x} \times n_{x}}$ represents the system matrix, each column of $\mathcal{B}_{u} \in$ $\mathbb{R}^{n_{x} \times n_{u}}$ maps each possible actuator control inputs to the system states, and each row of $\mathcal{C}_{y} \in \mathbb{R}^{n_{y} \times n_{x}}$ maps the system states to each possible sensor outputs. The matrix $\mathcal{B}_{w} \in \mathbb{R}^{n_{x} \times n_{w}}$ models the relation between the plant states and exogenous inputs such as external disturbances and 
references, $\mathcal{C}_{z} \in \mathbb{R}^{n_{z} \times n_{x}}$ between regulated output and the system states, $\mathcal{D}_{z w} \in \mathbb{R}^{n_{z} \times n_{w}}$ between regulated outputs and exogenous inputs, $\mathcal{D}_{z u} \in \mathbb{R}^{n_{z} \times n_{u}}$ between regulated outputs and system inputs and $\mathcal{D}_{y w} \in \mathbb{R}^{n_{y} \times n_{w}}$ between measured outputs and exogenous inputs.

The goal is to obtain a strictly proper dynamic output feedback controller $\mathcal{K}$ which is of the form:

$$
\mathcal{K}:\left\{\begin{array}{l}
\dot{\zeta}(t)=\mathcal{A}_{C} \zeta(t)+\mathcal{B}_{C} y(t) \\
u(t)=\mathcal{C}_{C} \zeta(t)
\end{array}\right.
$$

where $\zeta(t)$ represents the controller state vector. If the closed-loop transfer function from $w$ to $z$ is denoted by $\mathcal{T}$, then the closed-loop specifications $\mathcal{H}_{\infty}$ norm of $\mathcal{T}$ can be described as:

$$
\|\mathcal{T}\|_{\infty}=\sup _{\|w\|_{2} \neq 0} \frac{\|z\|_{2}}{\|w\|_{2}}
$$

where $\|w\|_{2}=\left[\int_{0}^{\infty} w^{T}(t) w(t) d t\right]^{1 / 2}$ is a 2-norm of a continuous signal. The basis of the combined design approach is the LMI formulations of the full order $\mathcal{H}_{\infty}$ control design are elaborated below without proofs (see Becker and Packard (1994) and Scherer et al. (1997) for proofs).

Lemma 1. There exists a feedback controller (2) that yields $\|\mathcal{T}\|_{\infty}<\gamma$ if, and only if there exists symmetric matrices $X, Y \in \mathbb{R}^{n_{x} \times n_{x}}$ and auxiliary control variables $\hat{\mathcal{A}}_{C} \in \mathbb{R}^{n_{x} \times n_{x}}, \hat{\mathcal{B}}_{C} \in \mathbb{R}^{n_{x} \times n_{y}}$ and $\hat{\mathcal{C}}_{C} \in \mathbb{R}^{n_{u} \times n_{x}}$ for which,

$$
\left[\begin{array}{c}
H e\left(\mathcal{A} X+\mathcal{B}_{u} \hat{\mathcal{C}}_{C}\right) \\
\hat{\mathcal{A}}_{C}+\mathcal{A}^{T} \\
\mathcal{B}_{w}^{T} \\
\mathcal{C}_{z} X+\mathcal{D}_{z u} \hat{\mathcal{C}}_{C}
\end{array}\right.
$$$$
\begin{gathered}
{ }^{\star} e\left(Y \mathcal{A}+\hat{\mathcal{B}}_{C} \mathcal{C}_{y}\right) \\
Y \mathcal{B}_{w}+\hat{\mathcal{B}}_{C} \mathcal{D}_{y w} \\
\mathcal{C}_{z}
\end{gathered}
$$$$
\left.\begin{array}{cc}
\star & \star \\
\star & \star \\
-I & \star \\
\mathcal{D}_{z w} & -\gamma^{2} I
\end{array}\right] \prec 0 \text { with }
$$

and

$$
\left[\begin{array}{cc}
X & I \\
I & Y
\end{array}\right] \succ 0
$$

The $\mathcal{H}_{\infty}$ feedback control optimization problem is formulated as

$$
\begin{aligned}
& \underset{X, Y, \hat{\mathcal{A}}_{C}, \hat{\mathcal{B}}_{C}, \hat{\mathcal{C}}_{C}, \gamma^{2}}{\operatorname{minimize}} \quad \gamma^{2} \\
& \text { subject to: }
\end{aligned}
$$

and the reconstruction is given as:

$$
\begin{aligned}
\mathcal{C}_{C}= & \hat{\mathcal{C}}_{C} R^{-T} \\
\mathcal{B}_{C}= & S^{-1} \hat{\mathcal{B}}_{C} \\
\mathcal{A}_{C}= & S^{-1}\left(\hat{\mathcal{A}}_{C}-\mathcal{B}_{C} \mathcal{D}_{y u} \hat{\mathcal{C}}_{C}-S \mathcal{B}_{C} \mathcal{C}_{y} X\right. \\
& \left.-Y \mathcal{B}_{u} \mathcal{C}_{C} R^{T}-Y \mathcal{A} X\right) R^{-T},
\end{aligned}
$$

where $R$ and $S \in \mathbb{R}^{n_{x} \times n_{x}}$ are nonsingular matrices which satisfies

$$
R S^{T}=I-X Y
$$

and can be obtained by singular value decomposition of $I-X Y$. Here, $\hat{\mathcal{A}}_{C}, \hat{\mathcal{B}}_{C}$ and $\hat{\mathcal{C}}_{C}$ are the auxiliary variables obtained when the linearizing change of variables is performed Becker and Packard (1994). The matrices $R$ and $S$ are not unique, however, they do not affect the closed-loop performance because the non-uniqueness of these matrices would only result in different realizations of the controller matrices( Scherer et al. (1997)).

Generally, $X$ and $Y$ are parameter dependent lyapunov functions for LPV feedback control design. However, there exists a case where the rates of the scheduling parameter are unbounded (and hence our assumption), when these symmetric matrices are considered parameter independent (refer Apkarian and Adams (2000)). In our formulations, the symmetric matrices $X$ and $Y$ are parameter independent. The LMI formulations can also be reformulated with parameter dependent matrices, when the bounds on the rates of scheduling parameter are considered finite. However, this introduces rational dependencies in the controller reconstruction (7), which can be avoided by adopting the approach for LPV controller reconstruction from Verbandt et al. (2018).

In case of the LPV systems, the system matrices have a piece-wise polynomial dependency on a multivariate scheduling parameter $\rho(t) \in \mathbb{R}^{n_{\rho}}$ that lies in the a prior known admissible compact convex set $\Lambda$. Furthermore, $\rho(t)$ is assumed to be continuously differentiable in the continuous-time. In this article, we consider a tensor product polynomial spline based parameterization (Hilhorst et al. (2016)). For a multivariate case, a parameter dependent matrix function is formulated as:

$$
S(\rho)=\sum_{i_{1}=1}^{n_{\lambda_{1}}-n_{d_{1}}-1} \cdots \sum_{i_{n_{\rho}}=1}^{n_{\lambda_{n_{\rho}}}-n_{d_{n_{\rho}}}-1} \mathbb{S}_{i_{1}, \ldots, i_{n_{\rho}}}\left(\mathfrak{B}_{i, n_{d}, \lambda}(\rho)\right)
$$

$$
\mathfrak{B}_{i, n_{d}, \lambda}(\rho)=\left(\prod_{k=1}^{n_{\rho}} \mathfrak{B}_{i_{k}, n_{d_{k}}, \lambda_{k}}\left(\rho_{k}\right)\right)
$$

where $n_{d}$ is the number of degrees, $\lambda \in \mathbb{R}^{n_{\lambda}}$ is a knot sequence, $\mathbb{S}_{i_{k}}\left(k=1 \ldots n_{\rho}\right)$ are the coefficient matrices and $\mathfrak{B}_{i_{k}, n_{d_{k}}, \lambda_{k}}(\rho)$ are the B-splines. Unique properties like positivity, partition of unity and minimal support are the advantages of using B-splines as basis to model the scheduling parameter dependencies.

\section{Big-M reformulation of a MBSDP problem}

This section deals with the combined problem of designing a dynamic output feedback controller of the form (2), considering the generalized plant (1), along with optimal selection of sensors and actuators.

\subsection{MBSDP formulation}

$\left\|\hat{\mathcal{C}}_{C}\right\|_{r-\ell_{0}}$ (and hence $\left\|\mathcal{C}_{C}\right\|_{r-\ell_{0}}$ ) represents the number of non-zero rows of $\hat{\mathcal{C}}_{C}$, where $r-\ell_{0}$ is the row $\ell_{0}$-quasi norm. The non-zero rows denotes the actuators to be used in the control design. Similarly, $\left\|\hat{\mathcal{B}}_{C}\right\|_{c-\ell_{0}}$ (and hence $\left\|\mathcal{B}_{C}\right\|_{c-\ell_{0}}$ ) denotes the number of non-zero columns of $\left\|\hat{\mathcal{B}}_{C}\right\|_{c-\ell_{0}}$ and thus the information on which sensors to be fed back. Thus, the mixed boolean semi-definite terms $\hat{B}_{C} \Phi$ and $\Psi \hat{C}_{C}$ for LTI systems and the terms $\hat{\mathbb{B}}_{C_{i_{k}}} \Phi$ and $\Psi \hat{\mathbb{C}}_{C_{i_{k}}}$ for LPV system determines the selection of sensors and actuators respectively, where $\hat{\mathbb{B}}_{C_{i_{k}}}$ and $\hat{\mathbb{C}}_{C_{i_{k}}}$ are the coefficients 
matrices of the B-spline functions $\hat{\mathcal{B}}_{C}$ and $\hat{\mathcal{C}}_{C}$ respectively, and $\Phi$ and $\Psi$ are defined as

$$
\Phi=\left[\begin{array}{ccc}
s_{1} & & 0 \\
& \ddots & \\
0 & & s_{n_{y}}
\end{array}\right], \Psi=\left[\begin{array}{ccc}
a_{1} & & 0 \\
& \ddots & \\
0 & & a_{n_{u}}
\end{array}\right] .
$$

to give $\Delta=\left\{s, a: s_{i}, a_{j} \in\{0,1\}\right\}$ which represents the set of all possible candidates of sensors and actuators and $s_{i}$ and $a_{j}$ as the boolean decision variables for $i^{t h}$ sensor and $j^{\text {th }}$ actuator respectively.

These mixed variables have a non-linear coupling which can be decoupled by applying Big-M reformulations on these variables transforming boolean constraints to a set of constraints that describe the same feasible set (see Luenberger et al. (1984)), which is shown by

$$
\begin{aligned}
& m_{B} \Phi \leqslant \hat{B}_{C} \leqslant M_{B} \Phi, \quad \Psi m_{C} \leqslant \hat{C}_{C} \leqslant \Psi M_{C}, \\
& m_{B} \Phi \leqslant \hat{\mathbb{B}}_{C_{i_{k}}} \leqslant M_{B} \Phi, \quad \Psi m_{C} \leqslant \hat{\mathbb{C}}_{C_{i_{k}}} \leqslant \Psi M_{C},
\end{aligned}
$$

for LTI systems and LPV systems respectively and $m_{B} \in \mathbb{R}_{-}^{n_{x} \times n_{y}}, M_{B} \in \mathbb{R}_{+}^{n_{x} \times n_{y}}, m_{C} \in \mathbb{R}_{-}^{n_{u} \times n_{x}}$ and $M_{C} \in$ $\mathbb{R}_{+}^{n_{u} \times n_{x}}$ are matrices representing Big-M bounds. If $M_{B}$ and $M_{C}$ are chosen sufficiently larger and $m_{B}$ and $m_{C}$ are chosen sufficiently smaller than any possible values of $\hat{B}_{C}$ or $\hat{\mathbb{B}}_{C_{i k}}$ and $\hat{C}_{C}$ or $\hat{\mathbb{C}}_{C_{i k}}$ respectively, then the Big-M reformulation is equivalent to the original constraint. Refer to appendix. A for a detailed insight.

\section{2 $\mathcal{H}_{\infty}$ controller synthesis with optimal selection of sensors and actuators}

Let there be additional requirements for $\left\{k: 1 \leqslant k \leqslant n_{y}\right\}$ sensors and $\left\{l: 1 \leqslant l \leqslant n_{u}\right\}$, which can be deduced in the following equality constraints,

$$
\operatorname{Tr}(\Phi)=k, \quad \operatorname{Tr}(\Psi)=l .
$$

The aim is to find the optimal set $\Delta$ such that it satisfies the desired closed loop specifications and satisfies the desired constraints for the number of sensors and actuators allocated for the control system design. This leads to a MBSDP optimization problem for the selection of the optimal set of $k$ sensors and $l$ actuators along with $\mathcal{H}_{\infty}$ feedback control design leading to the following theorem.

Theorem 2. The dynamic output feedback controller (2) is obtained for (1) that satisfies the closed-loop specification, $\|\mathcal{T}\|_{\infty}<\gamma$ and concurrently gives the optimal set of sensors and actuators, $\Delta$, for the desired $k$ sensors and l actuators as a solution to the optimization problem (14).

$$
\begin{array}{cl}
\underset{Y, \hat{\mathcal{A}}_{\mathcal{C}}, \hat{\mathcal{B}}_{\mathcal{C}}, \hat{\mathcal{C}}_{\mathcal{C}}, \gamma^{2}, s, a}{\operatorname{minimize:}} & \gamma^{2} \\
\text { subject to: } & (4),(5),(12),(13) .
\end{array}
$$

Refer appendix A for the proof.

Now, suppose we can assign implementation costs $\eta_{B}$ and $\eta_{C}$ as normalized costs for sensors and actuators respectively. The total implementation costs for the sensors and actuators can be denoted by $\operatorname{Tr}\left(\eta_{B} \Phi\right)$ and $\operatorname{Tr}\left(\eta_{C} \Psi\right)$. Here, $\eta_{B}$ and $\eta_{C}$ can be a scalar quantity, which would indicate that all the sensors or actuators have same costs or a diagonal matrix with distinct costs. This gives rise to a multi-objective optimization problem which is presented in the following theorem.

Theorem 3. The dynamic output feedback controller (2) is obtained for (1) that satisfies the closed-loop specification, $\|\mathcal{T}\|_{\infty}<\gamma$ and concurrently gives the optimal set of sensors and actuators, $\Delta$, that also minimizes their implementation costs as a solution to the optimization problem (15).

$$
\begin{array}{cl}
\underset{X, Y, \hat{\mathcal{A}}_{\mathcal{C}}, \hat{\mathcal{B}}_{\mathcal{C}}, \hat{\mathcal{C}}_{\mathcal{C}}, \gamma^{2}, s, a}{\operatorname{minimize:}} & \gamma^{2}+\operatorname{Tr}\left(\eta_{B} \Phi\right)+\operatorname{Tr}\left(\eta_{C} \Psi\right) \\
\text { subject to: } & (4),(5),(12) .
\end{array}
$$

Refer appendix A for the proof.

The Big-M reformulations in the MBSDP optimization problems (14) and (15) can be solved by a BNB algorithm to attain globally optimal solution. The results in this paper are obtained by the BNB algorithm of YALMIP (Lofberg (2004)). The BNB algorithm works by simultaneously building and searching a binary tree of optimization problems where every child is obtained from a parent via a branching procedure (see Morrison et al. (2016)).

\section{Application}

This section presents two applications to illustrate the capabilities of the presented MBSDP optimization. First, we considered an application of placing optimal actuators in a twenty-story building and design a $\mathcal{H}_{\infty}$ LTI feedback controller. Second, we consider a $\mathcal{H}_{\infty}$ LPV control design with optimal selection of collocated sensors and actuators of a smart composite plate.

\subsection{Vibration Control of a twenty-story building}

This subsection focuses on combined actuator selection with $\mathcal{H}_{\infty}$ feedback control design for the seismic isolation of tall buildings dwelled with a distributed set of actuators. It is assumed that the building stories are equipped with interstory force-actuation devices and sensing units that measures the interstory accelerations.

4.1.1 Modeling Description The building is modeled as depicted in Figure. 1, following the approach from PalaciosQuiñonero et al. (2018). The dynamical model for the lateral displacement can be constructed in state space matrices form as

$$
\begin{gathered}
\mathcal{A}=\Pi\left[\begin{array}{cc}
0_{n \times n} & I_{n} \\
-M^{-1} K_{s} & -M^{-1} C_{d}
\end{array}\right] \Pi^{-1}, \\
\mathcal{B}_{u}=\Pi\left[\begin{array}{c}
0_{n \times p} \\
\lambda M^{-1} T_{u}
\end{array}\right], \mathcal{B}_{w}=\Pi\left[\begin{array}{c}
0_{n \times 1} \\
-1_{n \times 1}
\end{array}\right]
\end{gathered}
$$

where $M$ and $K_{s}$ corresponds to the mass and stiffness matrices respectively that displays the mechanical characteristics of the building with $n$ stories and $p$ actuators and their 


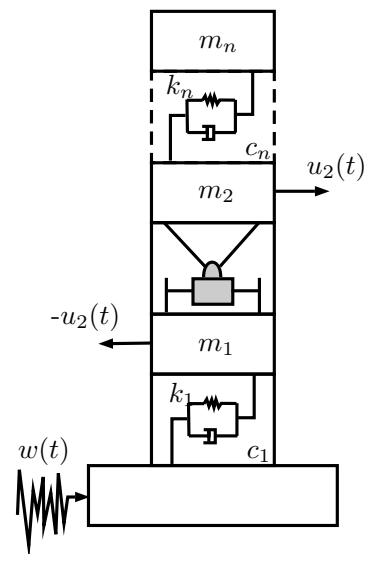

Figure 1. simplified mechanical model for a $n$-story building with masses $m_{i}$, stiffness $k_{i}$ and damping coefficients $c_{i}$ with interstory actuation devices which produce a pair of opposite structural forces.

structure is given as follows:

$$
\begin{gathered}
M=\left[\begin{array}{ccc}
m_{1} & & 0 \\
& \ddots & \\
0 & & m_{n}
\end{array}\right] \\
K_{s}=\left[\begin{array}{ccccc}
k_{1}+k_{2} & -k_{2} & & \\
-k_{2} & k_{2}+k_{3} & -k_{3} & & \\
& & \ddots & & \\
& & -k_{n-1} & k_{n-1}+k_{n} & -k_{n} \\
& & & -k_{n} & k_{n}
\end{array}\right]
\end{gathered}
$$

$(17)$

and the parameters $m_{i}$ and $k_{i}$ are given in Table. 1 and $\lambda=$ $6 \times 10^{4}$ in (17) represents a constant for scaling the inputs of the plant. Introducing this scaling factor does not affect the closed loop performance, but modifies the numerical model such that the minimum and maximum values of $\hat{C}_{C}$ in (7) do not affect the selection of Big-M constants for the combined control design and actuator selection problem. The selection of Big-M constant is also addressed in Section 5.

Table 1. Mass and stiffness parameters for the twenty-story building

\begin{tabular}{lcc}
\hline Story & Mass $\left(\times 10^{6} \mathrm{~kg}\right)$ & Stiffness $\left(\times 10^{8} \mathrm{~N} / \mathrm{m}\right)$ \\
\hline $1-5$ & 1.10 & 8.62 \\
$6-11$ & 1.10 & 5.54 \\
$12-14$ & 1.10 & 4.54 \\
$15-17$ & 1.10 & 2.91 \\
$18-19$ & 1.10 & 2.56 \\
20 & 1.10 & 1.72 \\
\hline
\end{tabular}

The damping matrix $C_{d}$ is chosen by considering relative damping of $2 \%$, i.e, $\zeta_{d}=0.02$ and is formulated using Rayleigh's damping method (See (Chopra 2001, p. 455)) with $C_{d}=a_{0} M+a_{1} K_{s}$ where $a_{0}$ and $a_{1}$ are the coefficients that are obtained by solving the following equation for $i^{t h}$ and $j^{t h}$ nodes, with $\omega$ as the frequency of the respective nodes

$$
\frac{1}{2}\left[\begin{array}{cc}
1 / \omega_{i} & \omega_{i} \\
1 / \omega_{j} & \omega_{j}
\end{array}\right]\left[\begin{array}{l}
a_{0} \\
a_{1}
\end{array}\right]=\left[\begin{array}{c}
\zeta_{d} \\
\zeta_{d}
\end{array}\right] .
$$

The control location matrix $T_{u}$ which specifies the structural effect of the actuation forces $u(t)$ is taken as:

$$
T_{u}=\left[\begin{array}{rrrr}
1 & -1 & & \\
& \ddots & \ddots & \\
& & 1 & -1 \\
& & & 1
\end{array}\right]
$$

and $w(t)$ is the disturbance created by seismic ground acceleration and $\Pi$ denotes the state transformation matrix such that

$$
x(t)=\Pi\left[\begin{array}{c}
q(t) \\
\dot{q}(t)
\end{array}\right]
$$

where $q(t)$ is the vector of story displacements with respect to the ground and finally, state vector $x(t)$ represents the interstory drifts.

4.1.2 Control Design The control configuration for seismic disturbance rejection control design for $n$-story building is shown in Figure. 2. with static loop-shaping weights $W_{D}$ and $W_{U}$, and $D$ is the disturbance channel.

In our case, $p=20$ and the weights are chosen as

$$
W_{D}=I_{20}, \quad W_{U}=\lambda 10^{-7.4} I_{20}
$$

to reduce the vibrations as low as possible (ideally $0 \mathrm{db}$ ) at each performance channels $\left(z_{1} \cdots z_{20}\right)$ and limit the actuator effort for the performance channels $\left(z_{u_{1}} \cdots z_{u_{20}}\right)$. Eventually, the control configuration and the weighting filters lead to the LTI generalized plant (1) producing the LTI dynamic output feedback controller (2) to form (6) which is solved using 'LCToolbox' *, an open source control design toolbox with in-built routines for MATLAB (See Verbandt et al. (2018)). The parsing of LMIs is done with YALMIP Lofberg (2004) and MOSEK Mosek (2015) to solve the SDP problem. The full-order controller is reconstructed using (7) which uses all the twenty interstory sensors and actuators.

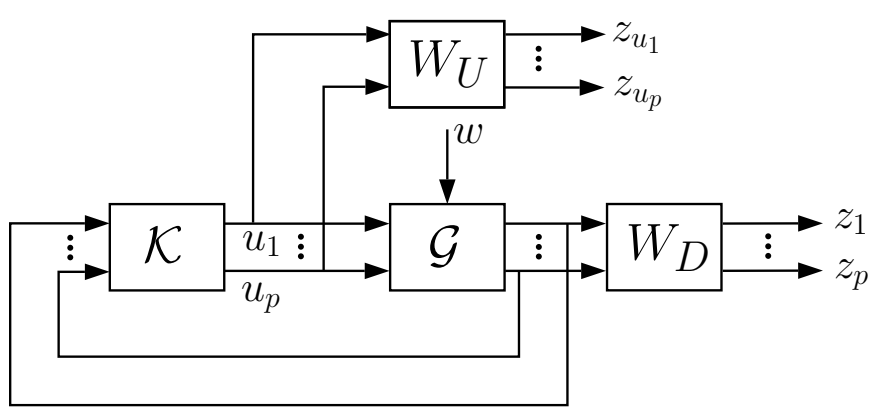

Figure 2. control configuration for disturbance rejection control design problem with $p$ actuators and sensors.

\subsubsection{Combined Control Design and Actuator Selection} Researchers Palacios-Quiñonero et al. (2018) investigated $\mathcal{H}_{\infty}$ state-feedback and partial state-feedback control design by allocating the actuator pair locations using three different strategies. The first strategy they considered was a concentrated actuation scheme by selecting the first ten

${ }^{*}$ https://github.com/meco-group/lc_toolbox 
locations. In the second strategy, they examined a semidistributed actuation scheme by selecting first five locations, and the rest five are distributed in the rest of the floors. In the third strategy, they imposed a distributed actuation scheme, selecting ten actuators on alternate floors. In order to select the optimal (optimal in terms of closed-loop performances) locations of the actuators we require to choose from ${ }^{20} C_{10}$ combinations. The number of combinations is reduced to ${ }^{16} C_{6}$, when an assumption is made that there are four known positions, which are common in all the above mentioned 3 schemes, viz; locations $1,3,5$ and 7 . The aim is to find the ten optimal locations for the actuators in the 20 -story building.

Firstly, the optimization problem (6) to obtain a fullorder $\mathcal{H}_{\infty}$ dynamic output feedback is solved by considering actuators in all 20 stories using 'LCToolbox'. The optimal $\gamma=2.1713$ represents an upper bound on the closed-loop performance for the control design. After obtaining the solution, the Big-M reformulation matrices $m_{C}$ and $M_{C}$ are constructed as follows

$$
m_{C}=10 \min \left(\hat{C}_{C}(:)\right), \quad M_{C}=10 \max \left(\hat{C}_{C}(:)\right) .
$$

where $\hat{C}_{C}(:)$ denotes all the elements $\hat{C}_{C}$. The optimization problem that attains the optimal solution for combined location and control design for the ten-story building is given by

$$
\begin{array}{cl}
\underset{X, Y, \hat{A}_{C}, \hat{B}_{C}, \hat{C}_{C}, \gamma_{\mathrm{lti}}^{2}, a}{\operatorname{minimize}} & \gamma_{\mathrm{lti}}^{2} \\
\text { subject to: } & (4),(5) \\
& \Psi m_{c} \leqslant \hat{C}_{C} \leqslant \Psi M_{C} \\
& \left\{a_{h}: h=1,3,5,7\right\}=1, \\
& \operatorname{Tr}(\Psi)=10,
\end{array}
$$

This optimization problem is parsed using YALMIP, and uses YALMIP's in-built BNB for solving the MBSDP problem and MOSEK for solving the SDP problems. The integer tolerance for $\mathrm{BNB}$ is chosen as intTol $=10^{-6}$. An additional test is carried out to check the sensitivity of the integer tolerance of the BNB tolerance for the formulation (23). As shown in Figure. 3, if the integer tolerance is smaller or larger, it does not affect convergence of the solution and it hardly affects the speed of convergence.
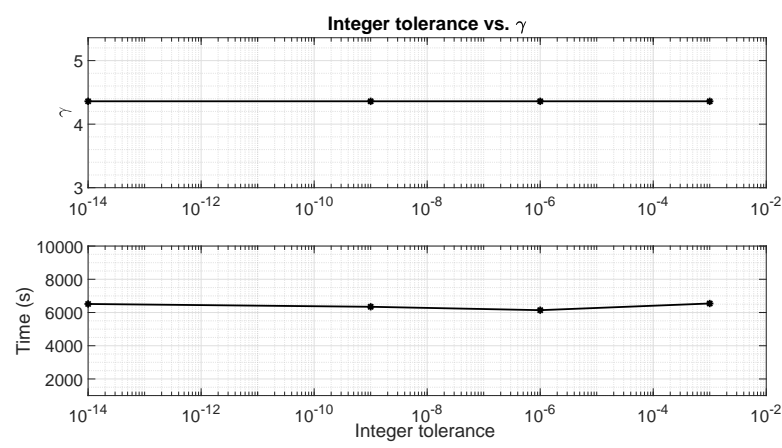

Figure 3. $\gamma$ and Computation time vs. Integer tolerance for Yalmip's BNB

The optimal actuator locations obtained corresponds to the concentrated scheme of selecting the first ten stories.
The optimal $\gamma_{\mathrm{lti}}$ achieved is 4.3598. After performing an ES by solving ${ }^{16} C_{6}$ combinations, it was found out that, the obtained solution by our approach is indeed globally optimal in terms of the actuator locations and also provides the exact closed loop performance cost.

We compared the computation time taken to find a solution using this approach with an ES for ${ }^{16} C_{6}$ combinations. The simulations were executed in MATLAB 2015b on a 4core (8 logical processors) with $2.70 \mathrm{GHz}$ Intel Core i7-820 CPU with 16GB RAM. For performing an ES, the control design problems were distributed parallel among 8 logical processors while only one processor was used for MBSDPBNB approach. It was found out that the computation time required to solve the combined problem using the mentioned approach $(1.8354 \mathrm{~h})$ is very less than the time required to obtain a solution using ES (12.8619h) even though the latter task was distributed.

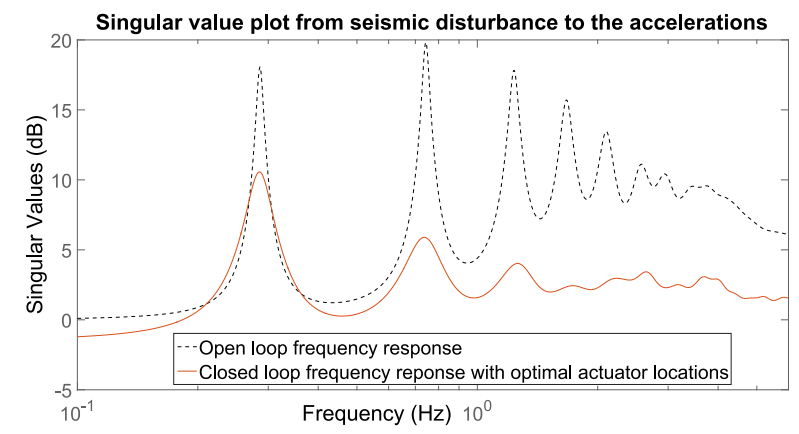

Figure 4. Singular value plot of the channel from disturbance $w$ to interstory accelerations $y_{1} \ldots y_{20}$ for comparing between the open loop response and closed loop response.
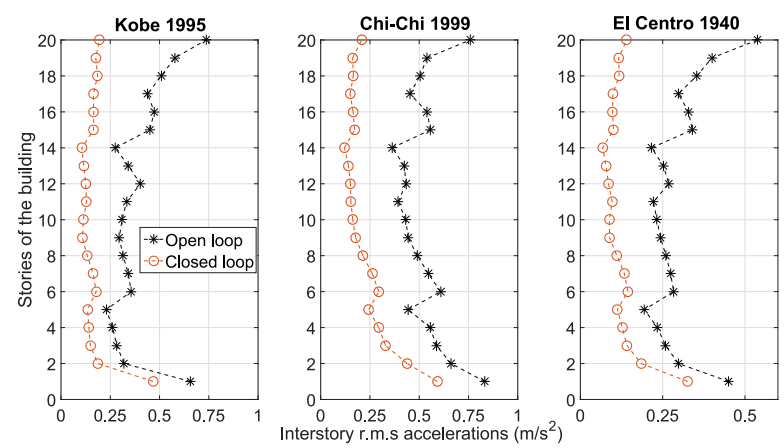

Figure 5. Interstory r.m.s accelerations obtained from time response from seismic ground disturbances $w$ of North-South Kobe 1995, Chi-Chi 1999 and El Centro 1940 for comparing between the open loop response and closed loop response.

After reconstructing the model (16) by eliminating the actuator positions at the last ten stories and with $\lambda=1$ that means without the input scaling, a controller is designed using the control configuration in Figure. 2. Figure. 4 represents the singular value responses of open loop and the closed loop from the disturbance $w$ to the all the 20 acceleration outputs. It can be easily seen that closed loop system adds a lot of damping at the resonances. Figure. 5 represents the time-domain analysis where the ground seismic disturbance profiles from three different places 
namely, El Centro 1940, North-South Kobe 1995 and ChiChi 1999 using 'OpenSeismoMATLAB' Papazafeiropoulos and Plevris (2018). The interstory r.m.s accelerations of 20 accelerations are recorded from open loop and closed loop and compared in the figure. Figure. 5 shows significant reduction in the r.m.s values of the accelerations.

\subsection{Active Vibration Control of a Composite Plate}

This subsection focuses on combined collocated sensor and actuator selection with $\mathcal{H}_{\infty}$ LPV control design for active vibration control of a composite plate.

4.2.1 Modeling Description The setup consists of a composite plate of dimension $503 \mathrm{~mm} \times 400 \mathrm{~mm} \times 2.5 \mathrm{~mm}$ composed of unidirectional carbon fiber laminates with a symmetric lay-up of $\left[-45^{\circ} 45^{\circ} 0^{\circ} 90^{\circ}\right]_{s}$ Dong et al. (2019). The plate is equipped with nine M2814-P1 macro-fiber composite (MFC) actuators of dimension $28 \mathrm{~mm} \times 14 \mathrm{~mm} \times$ $0.3 \mathrm{~mm}$ and with nine accelerometers. Force disturbances act on the plate at four locations, with their placements shown in Figure. 6. Since the weights of accelerometers are negligible (approx. 5 grams per unit) as compared to the actuators, the mechanical influence of sensors on the dynamics of the model is neglected.

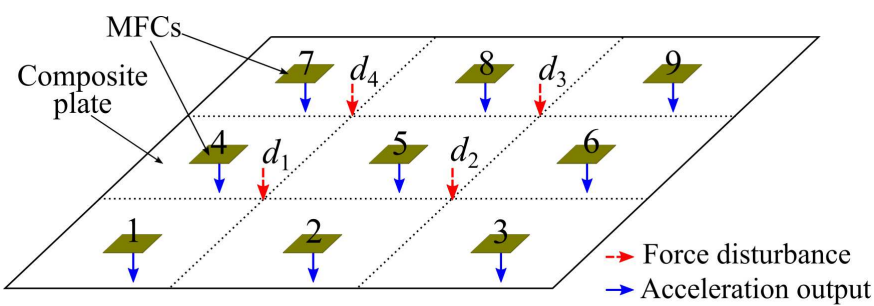

Figure 6. simplified model of a composite plate with $\left(d_{1} \ldots d_{4}\right)$ as the force disturbances applied to the plate.

Table 2. Structure and material parameters used in modeling of the composite plate.

\begin{tabular}{lcc}
\hline Parameter & MFC $-\mathbf{d 3 3}$ & laminates \\
\hline mass density & $5440 \mathrm{Kg} / \mathrm{m}^{3}$ & $1500 \mathrm{Kg} / \mathrm{m}^{3}$ \\
$Y_{11}$ & $27.142 \mathrm{GPa}$ & $110.15 \mathrm{GPa}$ \\
$Y_{22}$ & $14.8 \mathrm{GPa}$ & $7.868 \mathrm{GPa}$ \\
$G_{12}$ & $4.1312 \mathrm{GPa}$ & $4.05 \mathrm{GPa}$ \\
$\nu_{12}$ & 0.2922 & 0.26 \\
$G_{13}$ & $5.385 \mathrm{GPa}$ & - \\
$G_{23}=G_{31}$ & $10.5 \mathrm{GPa}$ & - \\
$d_{33}=d_{31}$ & $272 \mathrm{pm} / \mathrm{V}$ & - \\
$d_{32}$ & $-133 \mathrm{pm} / \mathrm{V}$ & - \\
$C_{p}$ & $0.30 \mathrm{nF} / \mathrm{cm}^{2}$ & - \\
\hline
\end{tabular}

To model the plate, we use their equivalent substrate modeling (ESM) approach Dong et al. (2019). The structural and material properties of the composite plate and MFC actuators are shown in the Table. 2, where $Y_{i j}$ are the Young's moduli, $G_{i j}$ are the shear moduli in $i j$ plane, $\nu_{i j}$ are the Poisson's ratios, $d_{i j}$ are piezoelectric charge constants and $C_{p}$ is the capacitance of the actuator. These parameters are used to obtain the dynamics of the plate with actuators Dong et al. (2017) and can be expressed as

$$
M \ddot{q}+C_{d} \dot{q}+\underbrace{\left(K_{s}-\Theta C_{p}^{-1} \Theta^{T}\right)}_{K_{a g}}=\Theta u+L w,
$$

where $q$ represents the displacement, $M, C_{d}$ and $K_{s}$ are the mass, damping and spring matrices respectively, $K_{a g}$ represents the augmented stiffness matrix which includes the piezoelectric induced stiffness and $\Theta$ is the piezoelectric coupling matrix. The localization matrix of disturbances is denoted by $L$, while $w=\left[d_{1}, d_{2}, d_{3}, d_{4}\right]^{T}$ and $u$ are the force disturbances and input voltage respectively. Second-OrderRational-Arnoldi approach Bai and $\mathrm{Su}$ (2005) is used to reduce the model (24) to five vibration modes resulting into the following tenth ${ }^{\text {th }}$ order state-space model

$$
\begin{aligned}
{\left[\begin{array}{c}
\dot{q} \\
\ddot{q}
\end{array}\right]=} & \underbrace{\left[\begin{array}{cc}
0 & I \\
-M_{r}^{-1} K_{r}^{a g} & -M_{r}^{-1} C_{r}
\end{array}\right]}_{A}\left[\begin{array}{c}
q \\
\dot{q}
\end{array}\right] \\
& +\underbrace{\left[\begin{array}{c}
0 \\
-M_{r}^{-1} \Theta_{r}
\end{array}\right]}_{B_{u}} u+\underbrace{\left[\begin{array}{c}
0 \\
-M_{r}^{-1} L_{r}
\end{array}\right]}_{B_{w}} w .
\end{aligned}
$$

where the matrices with subscript $(.)_{r}$ represents the respective matrices of the reduced-order model and the acceleration output $y$ is taken as,

$$
y=\left[\begin{array}{ll}
0 & C_{a}
\end{array}\right]\left[\begin{array}{c}
\dot{q} \\
\ddot{q}
\end{array}\right]
$$

such that substituting (25) into (26) results into

$$
y=\underbrace{\left[\begin{array}{ll}
0 & C_{a}
\end{array}\right] A}_{C}\left[\begin{array}{c}
q \\
\dot{q}
\end{array}\right]+\underbrace{\left[\begin{array}{ll}
0 & C_{a}
\end{array}\right]\left[\begin{array}{ll}
B_{u} & B_{w}
\end{array}\right]}_{D}\left[\begin{array}{c}
u \\
w
\end{array}\right] .
$$

The state-space model obtained is a LTI system. To illustrate the power and generality of the approach, an artificial LPV system is constructed by parameterizing the system matrix, $A$ with $\alpha$ as the artificially generated scheduling parameter, assuming values in the interval [0.05, 0.1], with $A(\alpha(t))=A+\alpha A$.

4.2.2 Control Design The control configuration for the force disturbance rejection for active vibration control of the composite plate is adopted as Figure. 2, where $w=\left[d_{1}, d_{2}, d_{3}, d_{4}\right]^{T}$ represents the force disturbances and $W_{D}$ and $W_{U}$ are the static loop-shaping weights for the disturbance channel $D$ and the input sensitivity channel $U$, respectively. The goal is to obtain a full-order dynamic output feedback LPV controller to minimize the cost function representing the closed-loop specification,

$$
\text { minimize: }\left\|\left[\begin{array}{l}
W_{D} D \\
W_{U} U
\end{array}\right]\right\|_{\infty} .
$$

The weights are selected as static gains as

$$
W_{D}=0.320 I_{9}, \quad W_{U}=10^{-2.25} I_{9} .
$$

to reduce the vibrations as low as possible as $10 \mathrm{db}$ at each performance channels $\left(z_{1} \ldots z_{9}\right)$ and limit the actuator effort for the performance channels $\left(z_{u_{1}} \ldots z_{u_{9}}\right)$. Using the 
system model constructed from (25) and (26) along with the weights (29) and attaining the control configuration results into a LPV dynamic output feedback controller which is solved using LCToolbox. The upper bound on the closed the closed-loop performance $\gamma_{\mathrm{lpv}}$ is 142.4256 . The slack variables $\hat{\mathcal{A}}_{\mathcal{C}}, \hat{\mathcal{B}}_{\mathcal{C}}$ and $\hat{\mathcal{C}}_{\mathcal{C}}$ are parameterized with B-Splines of degree 1 , and the knot sequence $[0.05,0.05,0.1,0.1]$. The parsing of the LMIs is done using YALMIP and OptiSpline Andersson (2013), Gillis et al. (2018), and MOSEK is used to solve the SDPs which results into a fullorder controller that uses all the sensors and actuators.

\subsubsection{Combined Control Design and Sensor/Actuator} Selection The main focus of this subsection is to attain a LPV output feedback controller combined with optimal sensor and actuator selection. After obtaining the solution to the control design problem as explained it in the previous subsection, the Big-M matrices are constructed using the following

$$
\begin{aligned}
m_{B_{1}} & =m_{B_{2}}=100 \min \left(\min \left(\hat{\mathbb{B}}_{C_{1}}(:)\right), \min \left(\hat{\mathbb{B}}_{C_{2}}(:)\right),\right. \\
M_{B_{1}}=M_{B_{2}} & =100 \max \left(\max \left(\hat{\mathbb{B}}_{C_{1}}(:)\right), \max \left(\hat{\mathbb{B}}_{C_{2}}(:)\right)\right. \\
m_{C_{1}} & =m_{C_{2}}=10 \min \left(\min \left(\hat{\mathbb{C}}_{C_{1}}(:)\right), \min \left(\hat{\mathbb{C}}_{C_{2}}(:)\right),\right. \\
M_{C_{1}}=M_{C_{2}} & =10 \max \left(\max \left(\hat{\mathbb{C}}_{C_{1}}(:)\right), \max \left(\hat{\mathbb{C}}_{C_{2}}(:)\right)\right.
\end{aligned}
$$

where $\hat{\mathbb{B}}_{C_{1}}(:), \hat{\mathbb{B}}_{C_{2}}(:)$ and $\hat{\mathbb{C}}_{C_{1}}(:), \hat{\mathbb{C}}_{C_{2}}(:)$ denotes all the elements in vector of the coefficient matrices of $\hat{\mathcal{B}}_{\mathcal{C}}$ and $\hat{\mathcal{C}}_{\mathcal{C}}$ respectively. The optimization that gives the optimal solution for the combined control design and collocated sensor/actuator selection is given by

$$
\begin{array}{cl}
\underset{X, Y, \hat{\mathcal{A}}_{C}, \hat{\mathcal{B}}_{C}, \hat{\mathcal{C}}_{C}, \gamma_{\mathrm{lpv}}^{2}, s, a}{\operatorname{minimize}} & \gamma_{\mathrm{lpv}}^{2}+\operatorname{Tr}\left(\eta_{C} \Psi\right) \\
\text { subject to: } & (4),(5),(12) \\
& 1<\operatorname{Tr}(\Psi)<n_{u_{\max }} . \\
& \Phi=\Psi .
\end{array}
$$

We assume that all the actuators cost are same, $\eta_{C}=1000$ and $n_{u_{\max }}=9$. Also, note that, the equality constraint $\Phi=$ $\Psi$ represents collocated sensor and actuator selection. This gives rise to 501 possible combinations distributed among the plate. The problem (31) is parsed using YALMIP and OptiSpline and solved using MOSEK and YALMIP's inbuilt BNB algorithm. The optimal sensor/actuator pair locations achieved are 1, 4, 6, 7 and 9. The upper bound on the closedloop performance is achieved as $\gamma_{\mathrm{lpv}}=147.1015$.

Figure. 7. shows the singular values of the LPV frequency response function matrix of the disturbances channels $d_{1} \ldots d_{4}$ to $y_{1} \ldots y_{9}$, evaluated at equidistant grid points of the scheduling parameter $\alpha$ of the disturbance channel. In the figure, we have the open loop singular value response $(\circ . \circ)$, the closed loop response with all the actuators and sensors (- - - -) and the closed loop response with an optimal selection of sensors and actuators (- - ). Both closed loop responses are well damped, with little differences between them. This shows that, although the number of actuators and sensors is reduced, the performance does not degrade substantially.

Figure. 8. represents the time-domain analysis. The force disturbances $d_{1} \ldots d_{4}$ are chosen as unit step disturbances while the scheduling parameter $\alpha$ is varied sinusoidal within

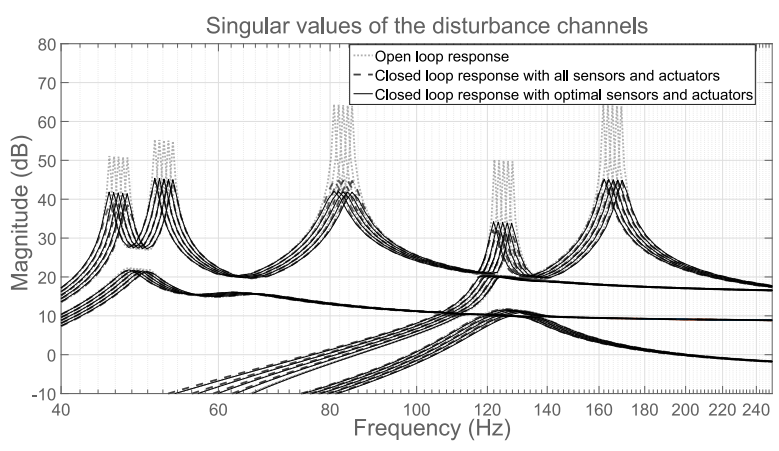

Figure 7. Singular value plot of the disturbance channel for comparing between the open loop response and closed loop responses.

its permissible range for generating the open and closed loop responses. In order to compare the open loop responses and closed loop responses, the r.m.s values of the accelerations obtained from the simulations. An average reduction of about $28 \%$ is achieved for the r.m.s values using this approach.

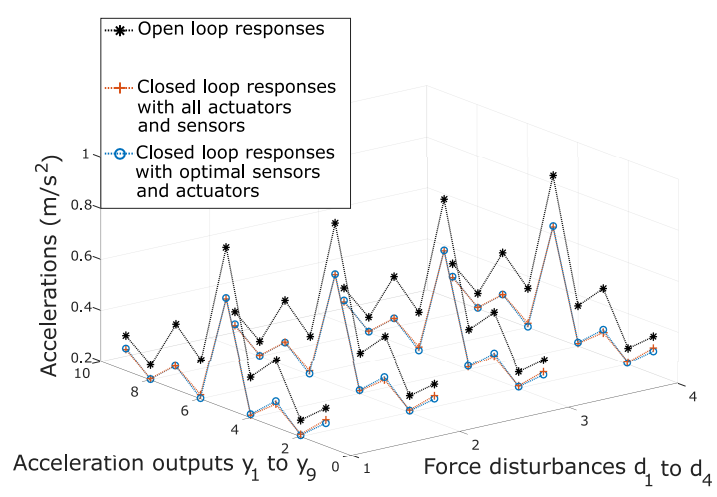

Figure 8. r.m.s accelerations obtained from time response from step force disturbances $d_{1} \ldots d_{4}$ for comparing between the open loop response and closed loop responses.

We also compared the computation time required to find an optimal solution using MBSDP approach with an ES of (31) for 501 combinations. The computation time using MBSDP is $0.02 \mathrm{~h}$, while using an ES is $0.95 \mathrm{~h}$. It is also found out that a globally optimal solution (globally optimal is terms of closed loop performance and sensor/actuator selection) is obtained.

\section{Comparison and limitations of combined design approaches}

In this section, we consider two LTI case studies with one of them already discussed in section. 4 to compare the MBSDP-BNB approach with three other approaches namely, IRLA Singh et al. (2018), MBSDP-OA Coey et al. (2018) and finally with an Exhaustive Search (ES).

For the combined control design with actuator selection using IRLA, we followed the approach from problem Singh et al. (2018). Here, we replace $\ell_{0}$ norm sparse function by iterative reweighted $\ell_{1}$ norm regularization Candes et al. (2008) in a multi-objective optimization. Here, the goal is 
to update the weights that are used for $\ell_{1}$-norm convex relaxation iteratively.

For combined control design using MBSDP-OA, we use the Pajarito solver, formulated in JuMP Dunning et al. (2017) in Julia programming language and use Gurobi Optimization (2014) and MOSEK as the sub solvers. Unlike Yalmip's BNB, which relaxes the integer constraints during the iterative BNB algorithm, MBSDPOA using Pajarito solver Coey et al. (2018) relaxes the SDP constraints. The underlying idea is to convert the SDP constraints into a finite-dimensional linear problem with linear constraints which are called cutting planes obtaining outer approximation of the original SDP problem.

The first case study is the seismic tall building rejection case as in section. 4. To apply IRLA Singh et al. (2018), three tuning parameters need to be set: the parameter $\eta_{C}$ that trades off the closed loop performance cost and the sparsity regularization, the convergence tolerance tol $=10^{-4}$, and a tuning parameter $\epsilon=10^{-5}$, to avoid singularities during the re-weighting process. The IRLA is formulated and solved and all results for this case study are summarized in table. 3. To apply MBSDP-OA, Pajarito solver requires duality gap tolerance for convergence, which was set as $10^{-5}$ and the results are shown in the table. 3. When compared with MBSDP-OA approach and ES, it was found out that MBSDP-BNB yield global optimal results for closed-loop performance and actuators selection, while IRLA achieves the global actuator selection however yields sub-optimal solution with respect to the closed loop performance.

MBSDP-BNB finds the global optimal solution about $1 / 7$ th of the time of the ES. The solution of IRLA closely approaches the global solution at a considerably shorter calculation time than all other approaches.

Table 3. Comparison of approaches for MIMO building system with 20 actuators.

\begin{tabular}{lccc}
\hline Approach & $\gamma$ & Global & Computation time $(\mathrm{h})$ \\
\hline IRLA & 4.3682 & $\times$ & 0.1576 \\
MBSDP-BNB & 4.3598 & $\checkmark$ & 1.8354 \\
MBSDP-OA & 5.9354 & $\times$ & 2.4709 \\
ES & 4.3598 & $\checkmark$ & 12.8619 \\
\hline
\end{tabular}

The second comparative case study is the vibration control design with optimal actuator selection of the composite plate for LTI system as discussed in section. 4. The difference lies in the grid of possible collocated sensor-actuator pairs, which is not a $5 \times 5$ grid instead of the $3 \times 3$ grid considered in section 5 , making this case more challenging. The tuning parameters for the IRLA are set to the same values as in the first comparative case study, except for $\eta_{C}=0.75$. The results are summarized in table. 4. For this case, only the MBSDP-BNB and of course the ES find the global optimal solution with IRLA and the MBSDP-BNB approach have comparable computation times. However, MBSDP-OA fails to converge to a global optimal solution.

The comparisons show that the approach followed in this article, i.e, MBSDP-BNB attain global optimal solution in terms of closed-loop performance and optimal selection which can be confirmed with ES. The computation time
Table 4. Comparison of approaches for MIMO composite plate system with 25 actuators.

\begin{tabular}{lccc}
\hline Approach & $\gamma$ & Global & Computation time $(\mathrm{h})$ \\
\hline IRLA & 165.4361 & $\times$ & 1.4416 \\
MBSDP-BNB & 112.8167 & $\checkmark$ & 1.5824 \\
MBSDP-OA & 115.4630 & $\times$ & 1.5278 \\
ES & 112.8167 & $\checkmark$ & 5.3739 \\
\hline
\end{tabular}

required to obtain the solution via MBSDP-BNB is however significantly less when compared with an ES.

IRLA is the fastest approach but fails to find the global solution in the second case study. which is probably caused by the relaxations of the sparsity inducing $\ell_{0}$ norm by a reweighted $\ell_{1}$ norm.

It is not guaranteed that MBSDP-OA converges to a global solution because a feasible solution to the linear programming approximation is not guaranteed to be a feasible solution to the semidefinite programming. An indepth analysis of this problem is out of the scope of this paper. In addition, since $\mathcal{H}_{\infty}$ control design is formulated in Matlab and Yalmip, and Pajarito requires the control design to be shifted to Julia and JuMP makes this method less convenient than using methods in Matlab.

Table. 5 summarizes the comparison of all the approaches that are used for combined control and selection design. The first two features mentioned in the table can be easily deduced from the results in the tables. 3 and 4. IRLA is not able to handle some of the boolean constraints, for example, the multi-objective problem (31) for achieving a trade-off between the closed-loop performance and total implementation cost of sensors and actuators. Additionally, translation of minimum and maximum actuator requirement in (31) into $\ell_{1}$-norm is not straightforward and requires a more detailed study, while the other approaches can handle such constraints.

Table 5. Summary of approaches for the combined design problems

\begin{tabular}{lcccc} 
Features & \multicolumn{4}{c}{ MBSDP- MBSDP- } \\
& & BNB & OA & \\
\hline $\begin{array}{l}\text { Global Optimal } \\
\text { Solution }\end{array}$ & $\times$ & $\checkmark$ & $\times$ & $\checkmark$ \\
\hline $\begin{array}{l}\text { Computationally } \\
\text { inexpensive }\end{array}$ & $\checkmark$ & $\checkmark$ & $\checkmark$ & $\times$ \\
\hline $\begin{array}{l}\text { Handle all } \\
\text { integer/boolean } \\
\text { constraints }\end{array}$ & $\times$ & $\checkmark$ & $\checkmark$ & $\checkmark$ \\
\hline $\begin{array}{l}\text { Determination of } \\
\text { Big-M constants }\end{array}$ & $\times$ & $\checkmark$ & $\checkmark$ & $\times$ \\
\hline
\end{tabular}

The main drawback of using MBSDP-BNB is the determination of the Big-M constants. In order to demonstrate the sensitivity of the solution to the Big-M constants, the numerical case of tall building is reconsidered but with a different boolean constraints than (23). The reason for considering a different problem is to reduce the number of combinations such that their computation time is reduced. 
The new problem formulation is given in (32)

$$
\begin{array}{cl}
\underset{Y, \hat{A}_{C}, \hat{B}_{C}, \hat{C}_{C}, \gamma_{\mathrm{lti}}^{2}, a}{\operatorname{minimize}} & \gamma_{\mathrm{lti}}^{2} \\
\text { subject to: } & (4),(5) \\
& \Psi m_{c} \leqslant \hat{C}_{C} \leqslant \Psi M_{C} \\
& \left\{a_{h}: h=1: 10\right\}=1, \\
& \operatorname{Tr}(\Psi)=12,
\end{array}
$$

where the actuators are fixed in first 10 stories and the objective is to select 2 out of the remaining 10 actuators which reduces the number of combinations from ${ }^{16} C_{6}$ to ${ }^{10} C_{2}$.

Clearly, since the control design specifications are same, the minimum and maximum values of $\hat{C}_{C}(:)$ are also same, and (22) can be written as:

$$
m_{C}=k \cdot \min \left(\hat{C}_{C}(:)\right), M_{C}=k \cdot \max \left(\hat{C}_{C}(:)\right)
$$

with $k=10$. The global solution obtained with exhaustive search is $\gamma=3.5822$ with the selected 2 actuators at the $11^{\text {th }}$ and $12^{\text {th }}$ story.
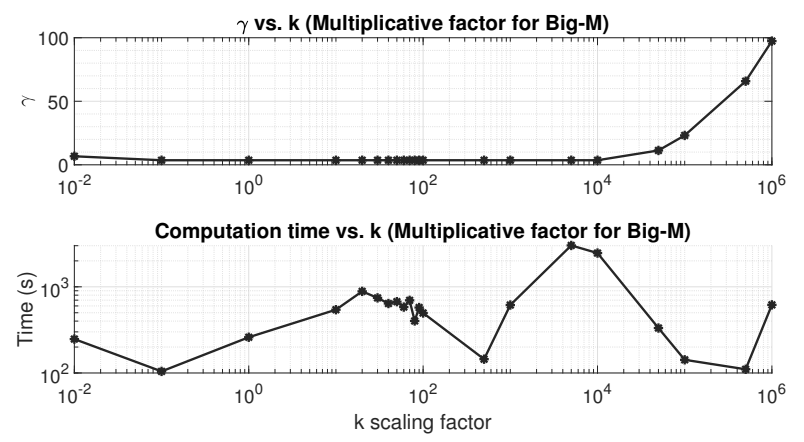

Figure 9. $\gamma$ and Computation time vs. k (Multiplicative factor for Big-M)

As shown in Figure. 9, when the scaling factor is significantly low, e.g $k<1$, the BNB is not able to find the global optimal solution. However, as we increase the scaling factor, the bounds on the matrix increases and it finally converges to the global optimal solution. With, respect to the computation time, the time increases with increase in the scaling factor, because the search domain for $\hat{C}_{C}$ is increased. Notice also in the bottom plot of Figure. 9, that the computation time does not linearly scale with the Big-M bounds.

If, the scalar factor is increased significantly, for, eg. $k \geq 10^{4}$, the algorithm does not converge to a global solution due to numerical problems that occur while solving the SDP optimization using Yalmip Lofberg (2004) and MOSEK Mosek (2015). In order to tackle this numerical problem, the primal-dual gap tolerance in MOSEK must be altered and by doing so, the SDP performance is relaxed, which is undesirable. In conclusion, a careful selection of Big-M constants is important.

\section{Conclusion}

In this article, we addressed combined feedback control design and optimal sensor/actuator selection using a MBSDP optimization formulation. The first contribution of our work lies in expressing the combinatorial optimization problem as a convex optimization problem by imposing Big-M constraints on the optimization variables of the controller. We introduced formulations for both LTI and LPV systems and proposed two different kind of optimization scheme for the combined control design and selection, theorem 2 and 3.

The second contribution of our article is to validate the proposed approach on two different applications: seismic rejection of a tall building and active vibration control of a composite plate. On comparing the singular value plots of the open loop and closed loop in both the applications, we found out that we were able to damp the resonances significantly even with a smaller number of sensors/actuators. Apart from that, we found out that there is a significant reduction in the r.m.s values of the open loop and closed loop responses in the disturbance channels.

The applications are compared with ES and another approaches: IRLA and MBSDP-OA. These case studies reveal the favorable properties of the developed approach: (i) calculation times significantly shorter than ES and similar as those of IRLA, and (ii) the global solution w.r.t to closedloop performance and optimal selection is attained.

\section{Declaration of Conflicting Interests}

The author(s) declared no potential conflicts of interest with respect to the research, authorship, and/or publication of this article.

\section{Funding}

The author(s) disclosed receipt of the following financial support for the research, authorship, and/or publication of this article: This work is supported by Flanders Make project: SBO ROCSIS: Robust and Optimal Control for Systems of Interacting Subsystems. This work also benefits from KU Leuven Research project C14/15/067: B-spline based certificates of positivity with applications in engineering.

\section{References}

Andersson J (2013) A general-purpose software framework for dynamic optimization. $\mathrm{PhD}$ Thesis, $\mathrm{PhD}$ thesis, Arenberg Doctoral School, KU Leuven, Department of Electrical Engineering (ESAT/SCD) and Optimization in Engineering Center, Kasteelpark Arenberg 10, 3001-Heverlee, Belgium.

Apkarian P and Adams RJ (2000) Advanced gain-scheduling techniques for uncertain systems. In: Advances in linear matrix inequality methods in control. SIAM, pp. 209-228.

Argha A, Su SW, Savkin A and Celler B (2019) A framework for optimal actuator/sensor selection in a control system. International Journal of Control 92(2): 242-260.

Argha A, Su SW, Savkin A and Celler BG (2017) Mixed $H_{2} / H_{\infty}$ based actuator selection for uncertain polytopic systems with regional pole placement. International Journal of Control : 117.

Bai Z and Su Y (2005) Dimension reduction of large-scale secondorder dynamical systems via a second-order arnoldi method. SIAM Journal on Scientific Computing 26(5): 1692-1709. 
Becker G and Packard A (1994) Robust performance of linear parametrically varying systems using parametricallydependent linear feedback. Systems \& Control Letters 23(3): 205-215.

Boyd S and Vandenberghe L (2004) Convex optimization. Cambridge university press.

Candes EJ, Wakin MB and Boyd SP (2008) Enhancing sparsity by reweighted $\ell_{1}$ minimization. Journal of Fourier analysis and applications 14(5-6): 877-905.

Chopra AK (2001) Dynamics of structures: theory and applications to earthquake engineering. Prentice-Hall.

Coey C, Lubin M and Vielma JP (2018) Outer approximation with conic certificates for mixed-integer convex problems. arXiv preprint arXiv: 1808.05290 .

Crusius CA and Trofino A (1999) Sufficient LMI conditions for output feedback control problems. IEEE Transactions on Automatic Control 44(5): 1053-1057.

De Oliveira MC and Geromel J (2000) Linear output feedback controller design with joint selection of sensors and actuators. IEEE Transactions on Automatic Control 45(12): 2412-2419.

Dhingra NK, Jovanović MR and Luo ZQ (2014) An ADMM algorithm for optimal sensor and actuator selection. In: Decision and Control (CDC), 2014 IEEE 53rd Annual Conference on. IEEE, pp. 4039-4044.

Dong Z, Faria C, Hromčík M, Pluymers B, Šebek M and Desmet W (2017) Equivalent force modeling of macro fiber composite actuators integrated into non-homogeneous composite plates for dynamic applications. Smart Materials and Structures 26(9): 095040

Dong Z, Faria C, Pluymers B, Hromčík M, Šebek M and Desmet W (2019) Structure-preserving low-order modeling approach of laminated composite plates integrated with macro-fiber composite transducers for dynamic applications. Composite Structures

Dunning I, Huchette J and Lubin M (2017) Jump: A modeling language for mathematical optimization. SIAM Review 59(2): 295-320.

Gahinet P and Apkarian P (1994) A linear matrix inequality approach to $\mathrm{H}_{\infty}$ control. International journal of robust and nonlinear control 4(4): 421-448.

Gawronski WK (2004) Dynamics and control of structures: A modal approach. Springer Science \& Business Media.

Gillis J, Lambrechts E and Pipeleers G (2018) Parametric and robust optimization with optispline. $37^{\text {th }}$ Benelux Meeting on Systems and Control

Goßmann F and Svaricek F (2019) Parameter dependent static output feedback control - an LPV approach. In: 2019 18th European Control Conference (ECC). pp. 3322-3327. DOI: 10.23919/ECC.2019.8795618.

Haemers M, Derammelaere S, Rosich A, Ionescu CM and Stockman K (2019) Towards a generic optimal co-design of hardware architecture and control configuration for interacting subsystems. Mechatronics 63: 102275.

Hilhorst G, Lambrechts E and Pipeleers G (2016) Control of linear parameter-varying systems using B-splines. In: Decision and Control (CDC), 2016 IEEE 55th Conference on. IEEE, pp. 3246-3251.

Hiramoto K, Doki H and Obinata G (2000) Optimal sensor/actuator placement for active vibration control using explicit solution of algebraic riccati equation. Journal of Sound and Vibration 229(5): 1057-1075.

Hiramoto K, Mohammadpour J and Grigoriadis KM (2009) Integrated design of system parameters, control and sensor/actuator placement for symmetric mechanical systems. In: Decision and Control, 2009 held jointly with the 2009 28th Chinese Control Conference. CDC/CCC 2009. Proceedings of the 48th IEEE Conference on. IEEE, pp. 2855-2860.

Lofberg J (2004) YALMIP: A toolbox for modeling and optimization in MATLAB. In: Computer Aided Control Systems Design, 2004 IEEE International Symposium on. IEEE, pp. 284-289.

Luenberger DG, Ye Y et al. (1984) Linear and nonlinear programming, volume 2. Springer.

Morrison DR, Jacobson SH, Sauppe JJ and Sewell EC (2016) Branch-and-bound algorithms: A survey of recent advances in searching, branching, and pruning. Discrete Optimization 19: 79-102.

Mosek A (2015) The mosek optimization toolbox for matlab manual.

Nestorović T and Trajkov M (2013) Optimal actuator and sensor placement based on balanced reduced models. Mechanical Systems and Signal Processing 36(2): 271-289.

Nugroho SA, Taha AF, Gatsis N, Summers TH and Krishnan R (2019) Algorithms for joint sensor and control nodes selection in dynamic networks. Automatica 106: 124-133.

Optimization G (2014) Inc., "Gurobi optimizer reference manual," 2015.

Palacios-Quiñonero F, Rubió-Massegú J, Rossell J and Rodellar J (2018) Design of distributed multi-actuator systems with incomplete state information for vibration control of large structures. Designs 2(1): 6.

Papazafeiropoulos G and Plevris V (2018) Openseismomatlab: A new open-source software for strong ground motion data processing. Heliyon 4(9): e00784.

Rubió-Massegú J, Rossell JM, Karimi HR and Palacios-Quinonero F (2013) Static output-feedback control under information structure constraints. Automatica 49(1): 313-316.

Sadabadi MS and Peaucelle D (2016) From static output feedback to structured robust static output feedback: A survey. Annual reviews in control 42: 11-26.

Scherer C, Gahinet P and Chilali M (1997) Multiobjective outputfeedback control via LMI optimization. IEEE Transactions on automatic control 42(7): 896-911.

Singh T, De Mauri M, Decré W, Swevers J and Pipeleers G (2018) Combined $\mathcal{H}_{\infty} / \mathcal{H}_{2}$ controller design and optimal selection of sensors and actuators. In: Joint $9^{\text {th }}$ IFAC Symposium on Robust Control Design and $2^{\text {nd }}$ IFAC Workshop on Linear Parameter Varying Systems. IFAC.

Singh T, Dong Z, De Mauri M, Decré W, Swevers J and Pipeleers G (2019) Combined $\mathcal{H}_{\infty}$ linear parameter varying control design and optimal sensor/actuator selection. In: 2019 18th European Control Conference (ECC). pp. 3310-3315. DOI: 10.23919/ECC.2019.8795969.

Singh T, Swevers J and Pipeleers G (2018) Concurrent $\mathcal{H}_{2} / \mathcal{H}_{\infty}$ feedback control design with optimal sensor and actuator selection. In: 2018 IEEE 15th International Workshop on Advanced Motion Control (AMC). pp. 223-228. DOI:10.1109/ AMC.2019.8371092. 
Summers TH, Cortesi FL and Lygeros J (2015) On submodularity and controllability in complex dynamical networks. IEEE Transactions on Control of Network Systems 3(1): 91-101.

Tropp JA (2006) Algorithms for simultaneous sparse approximation. part ii: Convex relaxation. Signal Processing 86(3): 589602.

Van De Wal M and De Jager B (2001) A review of methods for input/output selection. Automatica 37(4): 487-510.

Verbandt M, Jacobs L, Turk D, Singh T, Swevers J and Pipeleers G (2018) Linear Control Toolbox-supporting B-splines in LPV control. Mechatronics 52: 78-89.

\section{A Proof of theorems 2 and 3}

This appendix focuses on proving that the optimization in (14) and (15) obtain combined controller design with an optimal sensors/actuators selection by assigning optimal values to the boolean variables of the diagonal of $\Phi$ and $\Psi$. In order to do so, consider the following lemma:

Lemma 4. Given matrices $G, k, K \in \mathbb{R}^{n \times n}$ and a diagonal matrix $D \in \mathbb{R}^{n \times n}$, such that:

$$
k D \leqslant G \leqslant K D \quad(\text { or, similarly, } D k \leqslant G \leqslant D K),
$$

$$
\begin{aligned}
& \text { then, the following holds: }\|G\|_{c-\ell_{0}} \leqslant \\
& \|D\|_{c-\ell_{0}} \quad \text { or } \quad\left(\|G\|_{r-\ell_{0}} \leqslant\|D\|_{r-\ell_{0}}\right) \text {. }
\end{aligned}
$$

Proof. For proving by contradiction, assume that: $\|G\|_{c-\ell_{0}} \geq\|D\|_{c-\ell_{0}} \quad$ or $\quad\left(\|G\|_{r-\ell_{0}} \geq\|D\|_{r-\ell_{0}}\right)$, then, there exist at least a couple of indices $(r, c) \in\{1, \ldots, n\}^{2}$ such that:

$G^{(r, c)} \neq 0$ and $D^{(c, c)}=0 \quad$ or $\quad\left(G^{(r, c)} \neq 0\right.$ and $\left.D^{(r, r)}=0\right)$.

However, this contradicts the the condition (34) which implies that for any couple $(r, c) \in\{1, \ldots, n\}^{2}$ it holds:

$$
\begin{aligned}
& k^{(r, c)} D^{(c, c)} \leqslant G^{(r, c)} \leqslant K^{(r, c)} D^{(c, c)} \\
& \text { or } D^{(r, r)} k^{(r, c)} \leqslant G^{(r, c)} \leqslant D^{(r, r)} K^{(r, c)}
\end{aligned}
$$

Hence, using the lemma 4 and the binary nature of the variables used to define $\Phi$ and $\Psi$ we can conclude that,

$$
\begin{aligned}
& \left\|\hat{B}_{C}\right\|_{c-\ell_{0}} \leqslant\|\Phi\|_{c-\ell_{0}}=\operatorname{Tr}(\Phi) \\
& \text { and }\left\|\hat{C}_{C}\right\|_{r-\ell_{0}} \leqslant\|\Psi\|_{r-\ell_{0}}=\operatorname{Tr}(\Psi)
\end{aligned}
$$

This can be easily extended to the LPV counterpart by constructing B-splines. All the inequality constraints are imposed on the coefficients of the B-splines (9). By construction, B-splines are everywhere contained in the convex-hull of their coefficients or control points Hilhorst et al. (2016). Therefore, given the convexity of the elementwise constraints (12), we have

$m_{B} \Phi \leqslant \hat{B}_{C}(\rho) \leqslant M_{B} \Phi, \quad \Psi m_{C} \leqslant \hat{C}_{C}(\rho) \leqslant \Psi M_{C}, \quad \forall \rho \in \Lambda$,

Using, lemma 4. and (36), we can conclude,

$$
\begin{gathered}
\left\|\hat{B}_{C}(\rho)\right\|_{c-\ell_{0}} \leqslant\|\Phi\|_{c-\ell_{0}}=\operatorname{Tr}(\Phi) \\
\text { and }\left\|\hat{C}_{C}(\rho)\right\|_{r-\ell_{0}} \leqslant\|\Psi\|_{r-\ell_{0}}=\operatorname{Tr}(\Psi), \quad \forall \rho \in \Lambda
\end{gathered}
$$

where,

$$
\begin{aligned}
m_{B} & :=\left[\min _{i, k} m_{B_{i k}}^{(r, c)}\right]_{r, c}, M_{B}:=\left[\max _{i, k} M_{B_{i k}}^{(r, c)}\right]_{r, c}, \\
m_{C} & :=\left[\min _{i, k} m_{C_{i k}}^{(r, c)}\right]_{r, c}, M_{C}:=\left[\max _{i, k} M_{C_{i k}}^{(r, c)}\right]_{r, c} .
\end{aligned}
$$

